\title{
Criteria of the existence and of the associativity of the product of distributions
}

by

\section{J. MIKUSIŃSKI (Warszawa)}

Introduction. It is well known that the product $f(x) g(x)$ cannot be defined for all pairs of distributions $f(x)$ and $g(x)$. Moreorer, even when this product is defined, it fails to have the properties of the product of ordinary functions. For instance, the associativity law does not hold, in general. The aim of this paper is to give some simple criteria for the existence and for the associativity of the product of distributions. The definition of the product adopted here was giren in [1]. It is general enough and embraces the most important particular cases known.

1. Fundamental sequences and distributions. Indefinitely derivable functions will be called smooth functions. A sequence of smooth functions $\varphi_{n}(x)\left(x=\left(\xi_{1}, \ldots, \xi_{q}\right)\right)$ is fundamental in an open subset $O$ of the $q$-dimensional space if for every interval $I$ ( $q$-dimensional) whose closure is contained in $O$ there exist an order $k=\left(x_{1}, \ldots, x_{q}\right)$ and a uniformly convergent sequence of smooth functions $\Phi_{n}(x)$ such that

$$
\Phi_{n}^{(k)}(x)=\frac{\partial^{x_{1}+\ldots+\varkappa_{q}}}{\partial \xi_{1}^{\varkappa_{1}} \ldots \partial \xi_{q}^{\varkappa_{q}}} \Phi_{n}(x)=\varphi_{n}(x) .
$$

Two fundamental sequences $\varphi_{n}(x)$ and $\psi_{n}(x)$ are called equivalent in $O$ if the iterated sequence

$$
\varphi_{1}(x), \psi_{1}(x), \varphi_{2}(x), \psi_{2}(x), \ldots
$$

is fundamental in $O$. We then write $\varphi_{n}(x) \sim \psi_{n}(x)$.

Equivalence classes $\left[\varphi_{n}(x)\right]$ of sequences, equivalent in $O$, are called distributions and denoted by $f(x)$, as functions. In particular, every smooth function $\varphi(x)$ is a distribution and can be represented in the form $[\varphi(x)]$.

The above definition of distributions was assumed in [2] and [3].

2. Regular sequences. By a $\delta$-sequence we understand (see [3]) every sequence of non-negative smooth functions $\delta_{n}(x)$ with the following properties: 
(i) There exists a sequence of positive numbers $\alpha_{n}$, convergent to 0 , such that $\delta_{n}(x)$ vanishes outside the sphere $K_{n}: \xi_{1}^{2}+\ldots+\xi_{q}^{2} \leqslant \alpha_{n}^{2}$;

(ii) $\int_{-\infty}^{\infty} \delta_{n}(x) d x=1$, the integral being extended to the whole $q$-dimensional space.

Let us consider the sequence of convolutions

$$
\varphi_{n}(x)=f(x) * \delta_{n}(x)=\int_{-\infty}^{\sim} f(x-t) \delta_{n}(t) d t,
$$

where $f(x)$ is a distribution defined in an open set $O$. If $O$ is not identical with the whole $q$-dimensional space, the symbol ( 1 ) is meaningless. But we shall adopt the convention that the product $f(x-t) \delta_{n}(t)$ is defined in the whole space whenever the support $K_{n}$ of $\delta_{n}(x)$ is contained in the set of points where $f(x-t)$ is defined. We thus assume that that product vanishes outside $K_{n}$. By this corvention the functions $\varphi_{n}(x)$ are defined in the sets of the points whose distance from points outside $O$ is less than $2 \alpha_{n}$.

Given any distribution in 0 and any $\delta$-sequence $\delta_{n}(x)$, the sequence (1) will be called a regular sequence of $f(x)$. Every regular sequence of $f(x)$ is a fundamental sequence of $f(x)$.

3. Product of two distributions. By the product $f(x) g(x)$ of two distributions $f(x)$ and $g(x)$, defined in an open set $O$, we understand the distribution $\left[\varphi_{n}(x) \psi_{n}(x)\right]$, where $\varphi_{n}(x)$ and $\psi_{n}(x)$ are regular sequences of $f(x)$ and $g(x)$ respectively. The product is defined provided the sequence $\varphi_{n}(x) \psi_{n}(x)$ is fundamental. Such a definition is very general and embraces the most important cases.

The product just defined is not associative in general. This is shown by the well known example

$$
\left(\frac{1}{x} x\right) \delta(x)=\delta(x), \quad \frac{1}{x}(x \delta(x))=0,
$$

where $x$ is a real variable and $1 / x=(\log |x|)^{\prime}$. In order to have a condition which ensures associativity, it is convenient to employ the notion of order of a distribution.

4. Order of a distribution. We say that a distribution $f(x)$ is of order $k \geqslant 0$ in a $q$-dimensional open set $O$ if $f(x)$ is in $O$ the $k$-th derivative of a continuous function $f(x)$.

In the above definition, the coordinates $x_{i}$ of the order $k=\left(x_{1}, \ldots, x_{q}\right)$ are all non-negative integers. We also want to admit the case where all the coordinates $x_{i}$ are non-positive integers. We then say that $f(x)$ is of order $k$ in 0 if for every order $j$ such that $0 \leqslant j \leqslant-k$ the distribution $f^{(i)}(x)$ is a continuous function in $O$.
We shall also admit a more general case involving the preceding two. Let the coordinates of order $k$ be arbitrary integers. Denote by $k^{+}$ the order obtained from $k$ by replacing the negative coordinates of $k$ by 0 . Similarly, $k^{-}$will denote the order obtained from $k$ by replacing its positive coordinates by 0 . Evidently $k=k^{+}+k^{-}$. We say that $f(x)$ is of order $k$ in $O$ if there exists a continuous function $F(x)$ such that $F^{\left(k^{+}\right)}(x)=f(x)$ in $O$ and $F^{(j)}(x)$ is in $O$ a continuous function for $0 \leqslant$ $\leqslant j \leqslant-k^{-}$. This definition embraces the preceding two as particular eases.

If $f(x)$ is of order $k$ in $O$ and $\varphi_{n}(x)$ is its regular sequence, then for every interval $I$ whose closure is in $O$ there exist smooth functions $\Phi_{n}(x)$ such that $\Phi_{n}^{(k+)}(x)=\varphi_{n}(x)$ and the sequence $\Phi_{n}^{(j)}(x)$ converges uniformly in $I$ for $0 \leqslant j \leqslant-k^{-}$.

In fact, there is a continuous function $F(x)$ such that $F^{(k+)}(x)=f(x)$ and $F^{(j)}(x)$ is continuous for $0 \leqslant j \leqslant-k^{-}$. Letting

$$
\Phi_{n}(x)=F(x) * \delta_{n}(x)
$$

we have

$$
\text { 1) } \begin{aligned}
\Phi_{n}^{(k+)}(x)=F^{(k+)}(x) * \delta_{n}(x)=f(x) * \delta_{n}(x)=\varphi_{n}(x) & \\
\text { 2) }\left|\Phi_{n}^{(j)}(x)-F^{(j)}(x)\right| & =\left|\int_{-\infty}^{\infty}\left(F^{(j)}(x-t)-F^{(j)}(x)\right) \delta_{n}(t) d t\right| \\
\leqslant & \int_{-\infty}^{\infty}\left|F^{(j)}(x-t)-F^{(j)}(x)\right| \delta_{n}(t) d t
\end{aligned}
$$

and, for $0 \leqslant j \leqslant-k^{-}$, it follows from the continuity of $F^{(j)}(x)$ that $\Phi_{n}^{(j)}(x)$ converges to $F^{(j)}(x)$ uniformly in $I$.

5. An existence criterion for the product. We are going to prove the following criterion:

In order that the product $f(x) g(x)$ exist in $O$, it suffices that the distributions $f(x)$ and $g(x)$ be in $O$ of orders $k$ and $l$ such that $k+l \leqslant 0$.

Let $\varphi_{n}(x)$ and $\psi_{n}(x)$ be regular sequences of $f(x)$ and $g(x)$ respectively, and let $m=\max (k, l)$ (i. e. $\mu_{i}=\max \left(\varkappa_{i}, \lambda_{i}\right)$ for $k=\left(\varkappa_{1}, \ldots, \varkappa_{q}\right)$, $\left.l=\left(\lambda_{1}, \ldots, \lambda_{q}\right), m=\left(\mu_{1}, \ldots, \mu_{q}\right)\right)$. It suffices to prove the existence, for every interval $I$ whose closure is in $O$, of smooth functions $X_{n}(x)$ such that $X_{n}^{(m+)}(x)=\varphi_{n}(x) \psi_{n}(x)$ and that $X_{n}^{(j)}(x)$ converges uniformly in $I$ for $0 \leqslant j \leqslant-m^{-}$. It is easy to verify that this assertion holds when $k \leqslant 0$ and $l \leqslant 0$. To prove it generally, we proceed by induction. Let $e_{i}(1 \leqslant i \leqslant q)$ denote the order of which all the coordinates are 0 except the $i$-th coordinate, which is 1 . Suppose that the assertion holds for some $k^{+}, l^{+}$and for all $k^{-}, l^{-}$such that $k+l \leqslant 0$. We shall prove that if $\lambda_{i}+x_{i}$ 
$<0$, then the assertion holds when we replace $k$ by $k+e_{i}$ or $l$ by $l+e_{i}$. This will imply by induction that the assertion holds for all $k$ and $l$ such that $k+l \leqslant 0$. For reasons of symmetry it suffices to consider the case $k+e_{i}$ only. If $\varkappa_{i}<0$, then $\left(k+e_{i}\right)^{+}=k^{+}$, and there is nothing to prove. Suppose therefore that $x_{i} \geqslant 0$.

Let

$$
\chi_{n}(x)=\int_{\alpha_{i}}^{\xi_{i}} \varphi_{n}(t) d \tau_{i},
$$

where $\xi_{i}, \tau_{i}$ and $\alpha_{i}$ are the $i$-th coordinates of $x, t$ and $a$ respectively, $a$ being a fixed point in $I$. Evidently

$$
\varphi_{n} \psi_{n}=\left(\chi_{n}, \psi_{n}\right)^{\left(e_{i}\right)}-\chi_{n} \psi_{n}^{\left(e_{i}\right)} \text {. }
$$

The distributions $\left[\chi_{n}(x)\right],\left[\psi_{n}(x)\right]$ and $\left[\psi_{n}^{\left(e_{i}\right)}(x)\right]$ are of orders $k, l$, and $l+e_{i}$. Since $\varkappa_{i}+\lambda_{i}<0$ and $x_{i} \geqslant 0$, we have $\lambda_{i}<-1$ and $\left(l+e_{i}\right)^{+}=l^{+}$. Thus by the induction 'hypothesis, there exist smooth functions $\Phi_{n}(x)$ and $\Psi_{n}(x)$ such that

$$
\Phi_{n}^{(m+)}=\chi_{n} \psi_{n}(m=\max k, l), \quad \Psi_{n}^{(p+)}=\chi_{n} \psi_{n}^{\left(e_{i}\right)}\left(p=\max k_{i}, l+e_{i}\right)
$$

in $I$ and that $\Phi_{n}^{(j)}(x)$ converges almost uniformly for $0 \leqslant j \leqslant-m^{-}$ and $\Psi_{n}^{(j)}(x)$ converges almost uniformly for $0 \leqslant j \leqslant-p^{-}$. Since $\lambda_{i} \leqslant-1$, we have $m=p$. This implies that if

$$
X_{n}(x)=\Phi_{n}(x)+\int_{a_{i}}^{\xi_{i}} \Psi_{n}(t) d \tau_{i},
$$

then $X^{\left(m++e_{i}\right)}(x)=\varphi_{n}(x) \psi_{n}(x)$, by $(2)$, and $X_{n}^{(j)}(x)$ converges almost uniformly for $0 \leqslant j \leqslant-m^{-}$. Letting $r=\max \left(k+e_{i}, l\right)$, we have $r^{+}=$ $=m^{+}+e_{i}$ and $r^{-}=m^{-}$. Thus $X_{n}^{(r+)}(x)=\varphi_{n}(x) \psi_{n}(x)$ and $X_{n}^{(j)}(x)$ converges almost uniformly for $0 \leqslant j \leqslant-r^{-}$. Thus the induction inclusion is stated from $k$ to $k+e_{i}$, and the proof is achieved.

6. A criterion of associativity. In order to obtain a criterion of associativity for the product, we need a stronger form of the theorem proved in the foregoing section. Let us remark first that in the preceding proof it is not essential for $\varphi_{n}(x)$ and $\psi_{n}(x)$ to be regular. It suffices that there exist, for every interval $I$ whose closure is in $O$, smooth functions $\Phi_{n}(x)$ and $\bar{\Psi}_{n}(x)$ such that $\Phi_{n}^{(k+)}(x)=\varphi_{n}(x), \bar{\Psi}_{n}^{(l+)}(x)=\psi_{n}(x)$, and that $\Phi_{n}^{(j)}(x)$ converge uniformly in $I$ for $0 \leqslant j \leqslant-k^{-}$and $\overline{\Psi_{n}^{(j)}}(x)$ uniformly in $I$ for $0 \leqslant j \leqslant-l^{-}$.

Generally, let the sequence $\varphi_{n}(x)$ be called $k$-fundamental in $I$ for the distribution $f(x)$ if there are in $I$ smooth functions $\Phi_{n}(x)$ such that $\Phi_{n}^{(k+)}(x)=\varphi_{n}(x)$ and $\Phi_{n}^{(j)}(x)$ converges uniformly in $I$ for $0 \leqslant j \leqslant-k^{-}$. It follows from section 4 that if $f(x)$ is of order $k$ in $O$, then its every regular sequence is $k$-fundamental in every interval $I$ whose closure is
in $O$.
From the proof given in section 5 it follows that:

If, in $I, \varphi_{n}(x)$ is $k$-fundamental, $\psi_{n}(x)$ is l-fundamental and $k+l \leqslant 0$, then $\varphi_{n}(x) \psi_{n}(x)$ is m-fundamental, $m=\max (k, l)$.

- Evidently, if $\varphi_{n}(x)$ and $\bar{\varphi}_{n}(x)$ are $k$-fundamental for $f(x)$, then the interlaced sequence

$$
\varphi_{1}(x), \bar{\varphi}_{1}(x), \varphi_{2}(x), \bar{\varphi}_{2}(x), \ldots
$$

is also $k$-fundamental for $f(x)$. Similarly, if $\psi_{n}(x)$ and $\bar{\psi}_{n}(x)$ are $l$-fundamental for $g(x)$, then the sequence

$$
\psi_{1}(x), \bar{\psi}_{1}(x), \psi_{2}(x), \bar{\psi}_{2}(x), \ldots
$$

is $l$-fundamental for $g(x)$. Consequently, if $k+l \leqslant 0$, the sequence

$$
\varphi_{1}(x) \psi_{1}(x), \bar{\varphi}_{1}(x) \bar{\psi}_{1}(x), \varphi_{2}(x) \psi_{2}(x), \bar{\varphi}_{2}(x) \bar{\psi}_{2}(x), \ldots
$$

is $m$-fundamental. It represents the same distribution as each of the sequences $\varphi_{n}(x) \psi_{n}(x)$ and $\bar{\varphi}_{n}(x) \bar{\psi}_{n}(x)$. In particular, if $\bar{\varphi}_{n}(x)$ and $\bar{\psi}_{n}(x)$ are regular sequences, then $f(x) g(x)=\left[\bar{\varphi}_{n}(x) \bar{\psi}_{n}(x)\right]$ by the definition of the product, and consequently $f(x) g(x)=\left[\varphi_{n}(x) \psi_{n}(x)\right]$. This argument yields the following statement:

If, in $I, f(x)=\left[\varphi_{n}(x)\right], g(x)=\left[\psi_{n}(x)\right], \varphi_{n}(x)$ is k-fundamental, $\psi_{n}(x)$ is l-fundamental, and $k+l \leqslant 0$, then $f(x) g(x)=\left[\varphi_{n}(x) \psi_{n}(x)\right]$. tivity:

The last statement easily leads to the following criterion of associa-

Let $f(x), g(x)$ and $h(x)$ be distributions in $O$ of orders $k, l$ and $m$ respectively. If $k+l \leqslant 0, k+m \leqslant 0$, and $l+m \leqslant 0$, then

$$
(f(x) g(x)) h(x)=f(x)(g(x) h(x)) .
$$

In fact, let $\varphi_{n}(x), \psi_{n}(x)$ and $\chi_{n}(x)$ be regular sequences for $f(x), g(x)$ and $h(x)$ respectively. Then $\varphi_{n}(x)$ is $k$-fundamental, $\psi_{n}(x)$ is l-fundamental and $\chi_{n}(x)$ is $m$-fundamental. Since $k+l \leqslant 0$, the product $\varphi_{n}(x) \psi_{n}(x)$ is $\bar{m}$-fundamental, where $\bar{m}=\max (k, l)$. But the inequalities $k+m \leqslant 0$ and $l+m \leqslant 0$ imply that $\bar{m}+m \leqslant 0$. Thus the sequence $\left(\varphi_{n}(x) \psi_{n}(x)\right) \chi_{n}(x)$ is $\tilde{m}$-fundamental, $\tilde{n}=\max (\bar{m}, m)$. Consequently, both sides of the identity

$$
\left(\varphi_{n}(x) \psi_{n}(x)\right) \chi_{n}(x)=\varphi_{n}(x)\left(\psi_{n}(x) \chi_{n}(x)\right)
$$

are $\tilde{m}$-fundamental. Moreover, the product $\varphi_{n}(x) \psi_{n}(x)$ on the left side is $\bar{m}$-fundamental, and similarly, the product $\psi_{n}(x) \lambda_{n}(x)$ is $\bar{k}$-fundamental, where $\bar{k}=\max (l, m)$. Hence (4) implies $(3)$.

7. The order of the product. We shall prove that:

If $f(x)$ is of order $k$ and $g(x)$ of order $l$ in $O, k+l \leqslant 0$, then $f(x) g(x)$ is of order $m=\max (k, l)$ in $O$.

Studia Mathematica, xxr. 
From section 6 it follows only that $f(x) g(x)$ is of order $m$ in every interval whose closure is contained in an open set $O$, where $f(x)$ and $g(x)$ are of order $k$ and $l$. To have a general proof, we need the following statement:

If $f(x)$ is of order $k$ and $g(x)$ of order $l$ in $O, k+l+e_{i} \leqslant 0$, then the formula

$$
(f(x) g(x))^{\left(e_{i}\right)}=f^{\left(e_{i}\right)}(x) g(x)+f(x) g^{\left(e_{i}\right)}(x)
$$

holds in 0 .

In fact, if $\varphi_{n}(x)$ and $\psi_{n}(x)$ are regular sequences for $f(x)$ and $g(x)$, then $\varphi_{n}^{\left(e_{i}\right)}(x)$ and $\psi_{n}^{\left(e_{i}\right)}(x)$ are regular sequences for $f^{\left(e_{i}\right)}(x)$ and $g^{\left(e_{i}\right)}(x)$. Consequently, they are $\left(k+e_{i}\right)$-fundamental and $\left(l+e_{i}\right)$-fundamental respectively. This implies that the products $\varphi_{n}^{\left(e_{i}\right)}(x) \psi_{n}(x)$ and $\varphi_{n}(x) \psi_{n}^{\left(e_{i}\right)}(x)$ are fundamental and from the identity

$$
\left(\varphi_{n}(x) \psi_{n}(x)\right)^{\left(e_{i}\right)}=\varphi^{\left(e_{i}\right)}(x) \psi_{n}(x)+\varphi_{n}(x) \psi_{n}^{\left(e_{i}\right)}(x)
$$

the required formula is obtained.

The proof of the statement at the beginning of this section is similar to the proof from section 5. Remark first that the assertion is true when $k \leqslant 0$ and $l \leqslant 0$. To have a general proof, we proceed by induction. Suppose that the assertion holds for some $k^{+}, l^{+}$and for all $k^{-}, l^{-}$such that $z+l \leqslant 0$. We shall prove that if $\lambda_{i}+x_{i}<0$, then the assertion holds when we replace $k$ by $k+e_{i}$ or $l$ by $l+e_{i}$. This will imply by induction that the assertion holds for all $k$ and $l$ such that $k+l \leqslant 0$. For reasons of symmetry it suffices to consider the case $k+e_{i}$ only. If $x_{i}<0$, then $\left(k+e_{i}\right)^{+}=k^{+}$, and there is nothing to prove. Suppose therefore that $x_{i} \geqslant 0$.

There exist functions $F^{\prime}(x)$ and $G(x)$, continuous in $O$, such that $F^{\left(k^{+}+e_{i}\right)}(x)=f(x)$ and $G^{\left(l^{+}\right)}(x)=g(x)$. The distribution $F^{\left(k^{+}\right)}(x)$ is of order $k$ and the distribution $g^{\left(e_{i}\right)}(x)$ is of order $l+e_{i}$. Since $x_{i}+\lambda_{i}<0$ and $x_{i} \geqslant 0$, we have $\lambda_{i}<-1$ and $\left(l+e_{i}\right)^{+}=l^{+}$. By the induction hypothesis, the products $F^{\left(k^{+}\right)}(x) g(x)$ and $F^{\left(k^{+}\right)}(x) g^{\left(e_{i}\right)}(x)$ are of orders $m$ $=\max (k, l)$ and $p=\max \left(k, l+e_{i}\right)$, respectively. Since

$$
f(x) g(x)=\left(F^{(k+)}(x) g(x)\right)^{\left(e_{i}\right)}-F^{\left(k^{+}\right)}(x) g^{\left(e_{i}\right)}(x),
$$

it is evident that $f(x) g(x)$ is of order $r=\max \left(k+e_{i}, l\right)$ in $O$, and the proof is achieved.

8. A remark on the order of the sum. It is interesting to remark that a similar statement holds for the sum:

If $f(x)$ is of order $k$ and $g(x)$ of order $l$ in an open interval $I$, then $f(x)+g(x)$ is of order $m=\max (k, l)$ in $I$.

The proof is so easy that it is not worth reproducing here. It is interesting to note that the order of the sum is the same as the order of the product. Of course we need not suppose that $k+l \leqslant 0$. But the statement for the sum holds for an interval only and becomes false when we replace $I$ by an arbitrary open set $O$. In fact, the function $f(x)=\left(\xi_{1}^{2}+\xi_{2}^{2}\right)^{-1}$ is a distribution of order $k=(-\infty,-\infty)$ in the set $O$ of all the points of the plane except the origin. The distribution $g(x)=\delta\left(\xi_{1}\right)$ (delta distribution) is of order $l=(2,-\infty)$ in 0 . But their sum is not of order $m$ $=\max (k, l)=(2,-\infty)$.

9. An extension of the foregoing results. The criteria from sections 5 and 6 can be reinforced by introducing another definition of the order of a distribution. We shall say in this section that a distribution $f(x)$ is of order $k$ in $O$ if there exists a function $F^{\prime}(x)$ such that all its derivatives $F^{(j)}(x), 0 \leqslant j \leqslant-k^{-}$, are measurable functions in 0 , locally bounded, and that $F^{\left(k^{+}\right)}(x)=f(x)$.

If $f(x)$ is of order $k$ in $O$ and $\varphi_{n}(x)$ is its regular sequence, then for every interval $I$ whose olosure is in $O$ there exist smooth functions $\Phi_{n}(x)$ such that $\Phi_{n}^{\left(k^{+}\right)}(x)$ and the sequence $\Phi_{n}^{(j)}(x)$ is bounded in I and converges almost everywhere for $0 \leqslant j \leqslant-k^{-}$.

In fact, there exist a function $F^{\prime}(x)$ measurable and bounded, and its distributional derivatives $F^{(j)}(x), 0 \leqslant j \leqslant-k^{-}$, such that $F^{\left(k^{+}\right)}(x)$ $=f(x)$. The sequence $\Phi_{n}(x)=F(x) * \delta_{n}(x)$ has the required properties, which can be proved as in [1] (p. 168).

All the theorems from sections 5-8 remain true if the order of distributions is understood in the sense of this section. The proofs are the same and only need one modification namely that uniform convergent sequences should be replaced by bounded and almost everywhere convergent sequences.

\section{References}

[1] Jan Mikusiński, Irregular operations on distributions, Studia Math. 20 (1961), p. $163-169$.

[2] Jan Mikusiński and Roman Sikorski, The Elementary Theory of Distributions (I), Rozprawy Matematyezne 12 (1957).

[3] - The Elementary Theory of Distributions (II), Rozprawy Matematyczne 25 (1961).

Regu par la Rédaction le 2.3. 1961 